\title{
Tipranavir in the Protease Inhibitors Arena
}

\author{
Barbara Vergani and Stefano Rusconi \\ Università degli Studi di Milano, Dipartimento di Scienze Cliniche "Luigi Sacco", Sezione di Malattie \\ Infettive e Immunopatologia, Milan, Italy
}

Highly active antiretroviral therapy (HAART) has become the standard for treating HIV infection. ${ }^{[1]}$ HIV protease inhibitors (PIs) have been used as a major component of the HAART regimens over the past 10 years, along with nucleoside and non-nucleoside reverse transcriptase inhibitors. The introduction of these regimens resulted in an appreciable decline in morbidity and mortality due to HIV infection. ${ }^{[2,3]}$

Tipranavir (TPV) is a nonpeptidic PI, approved by the US FDA and the European Medicines Agency for the treatment of HIV infection. The structure of this molecule is a sulfonamidesubstituted dihydropyrone. Similar to other PIs, TPV binds directly to HIV aspartyl protease, disrupting the catalytic site of the enzyme and preventing protease-dependent cleavage of HIV gag and gag-pol polyproteins into smaller functional proteins ${ }^{[4-6]}$ For this reason, the chance to develop resistance is not so easy. TPV is active against PI multidrug resistant viruses and it has been very difficult to corner HIV to mutate through subsequent in vitro passages. ${ }^{[7-9]}$ TPV plus lowdose ritonavir $(\mathrm{TPV} / \mathrm{r})$ is used in combination with other antiretroviral (ARV) drugs. The advantages of this approach include raising trough drug concentrations, minimizing inter-patient variability, prolonging drug elimination half-life, and reducing pill burden. ${ }^{[10,11]}$ Results in the efficacy of the selected dose of TPV/r (500/200 mg twice daily) were quite encouraging in extensively treated HIVinfected patients. TPV is generally well tolerated; the most frequent adverse events were in the gastrointestinal system and laboratory abnormalities, such as alteration of the lipid profile and liver function. ${ }^{[12]}$

Darunavir (DRV) is another new-generation nonpeptidic HIV protease inhibitor approved by the FDA, used with low doses of ritonavir (600/100 mg twice daily) for pharmacologic enhancement (boosting). It has demonstrated potent activity against multidrug-resistant HIV in heavily treatment-experienced patients with HIV infection. Like TPV, DRV needs multiple mutations in the HIV protease for the virus to build a significant resistance to these drugs. For this reason, TPV and DRV exhibit a high genetic barrier to the emergence of novel resistant strains. ${ }^{[13,14]}$ Boosted DRV is generally well tolerated, with a lower incidence of diarrhea and modification of the lipid profile. ${ }^{[15]}$

The aim of the POTENT trial ${ }^{[16]}$ was the headto-head comparison of the safety and efficacy of TPV versus DRV plus a low dose of ritonavir, each with an optimized background regimen (OBR) in triple-class-experienced HIV-infected patients, being resistant to more than one PI.

This study was designed in 2007 (20 September) to enroll and treat $800 \mathrm{HIV}$-1-positive patients, randomly assigned $(1: 1)$ to one of the two arms: TPV/r (500/200 mg twice daily) or DRV/r (600/100 mg twice daily), with a treatment period of 50 weeks. Both drugs were administered in combination with another selected ARV, based on patient therapeutic history (and virtual phenotype screening results).

The major shortcoming of the POTENT trial was the very low enrollment numbers: only 39 patients (5\% of the planned number) were randomized and received drugs prior to early termination of the study (1 July 2008). The data from the trial, due to poor patient enrollment and premature termination, are insufficient to assess primary and secondary previously established endpoints. For this reason, statistical tests were not applied to this collected observational data. 
The demonstrations of effect of TPV/r have been more evident in treatment-experienced patients with HIV infection receiving an OBR including two fully active drugs. The use of enfuvirtide for the first time has been correlated with a higher rate of virologic success when combined with TPV/r. ${ }^{[12]}$ $\mathrm{TPV} / \mathrm{r}$ is generally well tolerated, nevertheless clinical hepatitis and hepatic decompensation have been associated with its use. Although initially reported, a recent case review from the FDA did not identify new safety concerns regarding intracranial hemorrhage. Hepatic evaluation is highly recommended in co-infected patients who are at risk for hepatic decompensation. ${ }^{[17,18]}$

There is evidence that mutations conferring resistance to TPV are not related to resistance to DRV. This may be important for the potential sequencing of these two PI in multiple ARVexperienced patients. A substantial susceptibility to TPV and DRV of viral isolates derived from patients with a massive ARV drug experience has been reported. ${ }^{[19]}$ In multiple ARV-experienced patients with resistance to one or more PI, TPV and DRV remain the most likely active PIs for constructing an effective drug regimen.

Nowadays, mostly achievable goals of ARV are to further reduce HIV-related morbidity and mortality, improve quality of life, and restore and preserve immunologic function in HIV-infected individuals through a sustained suppression of HIV replication. ${ }^{[20,21]}$

It is not so easy to design a trial such as POTENT where even after significant efforts to enroll patients, the number of individuals included in the study and time in the follow-up were insufficient to draw definitive conclusions. Furthermore, the POTENT trial included a comparison between two PIs; however what we really need are combination regimens including drugs with new mechanisms of action, new cellular targets and novel chemical structures (e.g. coreceptor or integrase inhibitors), together with an improved tolerability profile. ${ }^{[22]}$

\section{Acknowledgments}

The authors have no conflicts of interest to declare that are directly relevant to the content of this article.

\section{References}

1. Thompson MA, Aberg JA, Cahn P, et al. Antiretroviral treatment of adult HIV infection: 2010 recommendations of the International AIDS Society-USA Panel. JAMA 2010; 304: 321-33

2. Palella FJ, Delaney KM, Moorman AC, et al. Declining morbidity and mortality among patients with advanced human immunodeficiency virus infection. HIV Outpatient Study Investigators. N Engl J Med 1998; 338: 853-60

3. Patick AK, Potts KE. Protease inhibitors as antiviral agents Clin Microbiol Rev 1998; 11: 614-27

4. Turner SR, Strohbach JW, Tommasi RA, et al. Tipranavir (PNU-140690): a potent, orally bioavailable non-peptidic HIV protease inhibitor of the 5,6-dihydro-4-hydroxy-2pyrone sulfonamide class. J Med Chem 1998; 41: 3467-76

5. Thiasrivongs S, Strohbach JW. Structure-based discovery of tipranavir disodium (PNU-140690E): a potent, orally bioavailable, nonpeptidic HIV protease inhibitor. Biopolymers 1999; 51: 51-8

6. Monini P, Sgadari C, Barillari G, et al. HIV protease inhibitors: antiretroviral agents with anti-inflammatory, antiangiogenic and anti-tumor activity. J Antimicrob Chemother 2003; 51: 207-11

7. Rusconi S, La Seta Catamancio S, Citterio P, et al. Susceptibility to PNU-140690 (tipranavir) of human immunodeficiency virus type 1 isolates derived from patients with multidrug resistance to other protease inhibitors. Antimicrob Agents Chemother 2000; 44: 1328-32

8. Back NKT, van Wijk A, Remmerswaal D, et al. In-vitro tipranavir susceptibility of HIV-1 isolates with reduced susceptibility to other protease inhibitors. AIDS 2000; 14 : 101-2

9. Larder BA, Hertogs K, Bloor S, et al. Tipranavir inhibits broadly protease inhibitor-resistant HIV-1 clinical samples. AIDS 2000; 14: 1943-8

10. Moyle G. Use of HIV protease inhibitors as pharmacoenhancers. AIDS Read 2001; 11: 87-98

11. Cooper CL, van Heeswijk RP, Gallicano K, et al. A review of low-dose ritonavir in protease inhibitor combination therapy. Clin Infect Dis 2003; 36: 1585-92

12. Hicks CB, Cahn P, Cooper DA, et al. Durable efficacy of tipranavir-ritonavir in combination with an optimised background regimen of antiretroviral drugs for treatmentexperienced HIV-1-infected patients at 48 weeks in the Randomized Evaluation of Strategic Intervention in multidrug reSistant patients with Tipranavir (RESIST) studies: an analysis of combined data from two randomised openlabel trials. Lancet 2006; 368: 466-75

13. de Meyer S, Vangeneugden T, van Baelen B, et al. Resistance profile of darunavir: combined 24-weeks results from the POWER trials. AIDS Res Hum Retroviruses 2008; 24: $379-88$

14. De Luca A. Resistance to newly approved and investigational protease inhibitors. Curr Opin HIV AIDS 2007; 2: 130-6

15. Arastéh K, Yeni P, Pozniak A, et al. Efficacy and safety of darunavir/ritonavir in treatment-experienced HIV type-1 patients in the POWER 1, 2 and 3 trials at week 96. Antivir Ther 2009; 14: 859-64

16. Elgadi MM, Piliero PJ. Boosted tipranavir versus darunavir in treatment-experienced patients: observational data from 
the randomized POTENT trial. Drugs R D 2011; 11 (4): 295-302

17. Di Vincenzo P, Carosi G, Angarano G, et al. Gammaglutamyltranspeptidase (gamma-GT) levels before treatment are predictive of tipranavir interruption in ART multiexperienced HIV-1-infected patients. HAART and Correlated Pathologies 2008; 1: 7-12

18. Macìas J, Orihuela F, Rivero A, et al. Hepatic safety of tipranavir plus ritonavir (TPV/r)-based antiretroviral combinations: effect of hepatitis co-infection and pre-existing fibrosis. J Antimicrob Chemother 2009; 63: 178-83

19. Baxter JD, Shapiro JM, Boucher CA, et al. Genotypic changes in human immunodeficiency virus type 1 protease associated with reduced susceptibility and virologic response to the protease inhibitor tipranavir. J Virol 2006; 21: 10794-801

20. Panel on Antiretroviral Guidelines for Adults and Adolescents. Guidelines for the use of antiretroviral agents in
HIV-1-infected adults and adolescents. Department of Health and Human Services. January 10, 2011; 1-166 [online]. Available from URL: http://www.aidsinfo.nih.gov/ ContentFiles/AdultandAdolescentGL.pdf [Accessed 2011 Aug 1]

21. Currier J, Baden L. Getting smarter: the toxicity of undertreated HIV infection. N Engl J Med 2006; 355: 2359-61

22. Moyle G, Gatell J, Perno C-F, et al. Potential for new antiretrovirals to address unmet needs in the management of HIV-infection. AIDS Patient Care STDs 2008; 22: 459-71

Correspondence: Dr Stefano Rusconi, Università degli Studi di Milano, Dipartimento di Scienze Cliniche "Luigi Sacco", Sezione di Malattie Infettive e Immunopatologia, Via G.B. Grassi, 74, 20157 Milan, Italy.

E-mail: stefano.rusconi@unimi.it 